\title{
BeiDou Satellites Assistant Determination by Receiving Other GNSS Downlink Signals
}

\author{
Lei Chen, ${ }^{1}$ Ke Zhang, ${ }^{1}$ Xiangwei Zhu, ${ }^{1}$ Yangbo Huang, ${ }^{1}$ Gang Ou, ${ }^{1}$ and Huicui Liu ${ }^{2}$ \\ ${ }^{1}$ College of Electronic Science and Engineering, National University of Defense Technology, Changsha 410073, China \\ ${ }^{2}$ National Key Laboratory of Science and Technology on Aerospace Flight Dynamics, Beijing Aerospace Control Center, \\ Beijing 100094, China \\ Correspondence should be addressed to Lei Chen; chenlei1025@nudt.edu.cn
}

Received 2 March 2016; Accepted 2 June 2016

Academic Editor: Stefania Bonafoni

Copyright (C) 2016 Lei Chen et al. This is an open access article distributed under the Creative Commons Attribution License, which permits unrestricted use, distribution, and reproduction in any medium, provided the original work is properly cited.

\begin{abstract}
GNSS's orbit determinations always rely on ground station or intersatellite links (ISL). In the emergency of satellite-to-ground links and ISL break-off, BeiDou navigation satellite system (BDS) satellites cannot determine their orbits. In this paper, we propose to add a spaceborne annular beam antenna for receiving the global positioning system (GPS) and global navigation satellite system (GLONASS) signals; therefore, the BDS satellites may be capable of determining their orbits by GPS/GLONASS signals. Firstly, the spectrum selection, the power isolation, the range of Doppler frequency shift, and changing rate are taken into account for the feasibility. Specifically, the L2 band signals are chosen for receiving and processing in order to prevent the overlapping of the receiving and transmitting signals. Secondly, the minimum number of visible satellites (MNVS), carrier-to-noise ratio $\left(C / N_{0}\right)$, dilution of precision (GDOP), and geometric distance root-mean-square (gdrms) are evaluated for acquiring the effective receiving antennas' coverage ranges. Finally, the scheme of deploying 3 receiving antennas is proved to be optimal by analysis and simulations over the middle earth orbit (MEO), geostationary earth orbit (GEO), and the inclined geosynchronous satellite orbit (IGSO). The antennas' structures and patterns are designed to draw a conclusion that installing GPS and GLONASS receivers on BDS satellites for emergent orbits determination is cost-effective.
\end{abstract}

\section{Introduction}

With the rapid developing of the global navigation satellite system (GNSS), satellites' orbit determination becomes a problem as it can only be measured from ground-station observing systems (GSOS) including the United X-band tracking telemetering and control system (UXB) and very long baseline interferometry (VLBI). The predicted orbital parameters can be injected into the satellites by a huge terrestrial uploading antenna. For the satellites beyond the ground-station observing systems' tracking telemetry and command (TT\&C) ranges, the intersatellite links (ISL) established on BeiDou navigation satellite system (BDS) or global positioning system (GPS) can be used for relay transmissions.

However, GSOS are complex and expensive to be maintained, while ISL is susceptible to jamming, which prevents the GNSS from continuing broadcast accurate navigation signals. To possess the ability of orbit determination without supporting by the GSOS and ISL, we propose to install onboard GNSS receiver on BDS satellites for receiving navigation signals from other GNSSs. By receiving navigation signals from GPS, Galileo, or global navigation satellite system (GLONASS), the BDS satellites' orbit may be determined in order to ensure the accuracy of the broadcasted BDS signal as well as maintaining other functions uninterruptedly. The proposed scheme, BDS orbit determination by GPS/GLONASS (BODGG), requires simpler maintenance, lower cost than the GSOS/ISL solutions. Also, the BODGG scheme is less influenced by the atmosphere (error caused by troposphere and ionosphere).

The spaceborne GPS receivers have been widely used on low earth orbit (LEO) spacecraft, where the height of LEO is lower than the height of GPS's orbit. GPS signals transmit towards the earth so that the LEO spacecrafts can easily receive the signals for positioning [1]. While the high earth orbit (HEO) spacecrafts such as the geostationary 


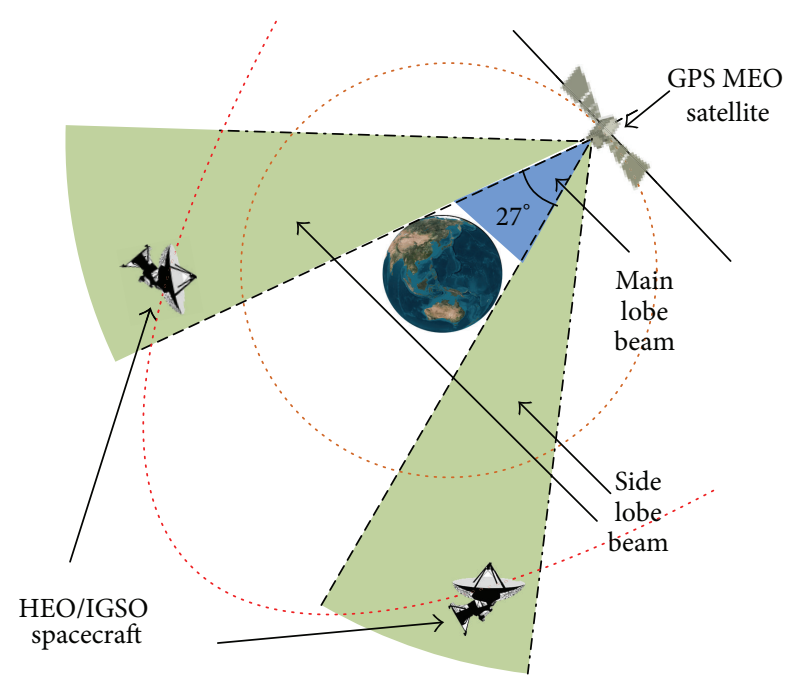

FIGURE 1: Diagrammatic sketch of GPS and HEO/IGSO satellites' position.

earth orbit (GEO) satellites and the inclined geosynchronous satellite orbit (IGSO) satellites can hardly receive GPS's or others GNSS's downlink signals since they are higher than the navigation satellites, and a solution is to receive the leaked signals from the side lobe beams of the GPS satellite's transmitting antennas. As shown in Figure 1, the above solution is based on the fact that the GPS antenna contains a main lobe beam towards the earth and a side lobe beam towards the space around the earth. However, a challenge is brought about as the signals emitted from the side lobe may be too weak to be effectively utilized by the HEO satellites [2].

In order to address the above challenges, Moreau and Christian have analyzed the visibility of GNSS satellites observed from GEO [3,4]. Qiao et al. have calculated the geometric dilution of precision (GDOP) of the received GNSS signals on GEO satellites [5]. However, the solutions in [3-5] did not take into account the fact that a GNSS satellite needs to simultaneously transmit navigation signals to provide localization services as well as receiving navigation signals for locating itself. Therefore, the second challenge to be dealt with is to support simultaneously receiving GNSS signals and transmitting BDS signals, since the receivers installed on BDS satellites may be easily interfered by the downlink signals transmitted from themselves.

Against the background, we propose to address the above-mentioned two challenges in this paper, where the BDS satellites operate on GEO, IGSO, and MEO; the feasibility of GNSS side lobe signals received by BDS satellites on the 3 orbits is analyzed. The rest is organized as follows. In Section 2, the appropriate receivable signals are chosen, and the isolation budget is calculated. In Section 3, the signal receiving performance is analyzed by evaluating the Doppler frequency shift $\left(f_{\mathrm{d}}\right)$, the Doppler changing rate $\left(d f_{\mathrm{d}} / d t\right)$, the minimum number of visible satellites (MNVS), the carrier-to-noise ratio $\left(C / N_{0}\right)$, the GDOP, and the geometric distance root-mean-square (gdrms). In Section 4, the optimal antenna beamwidth and elevation are obtained numerically.

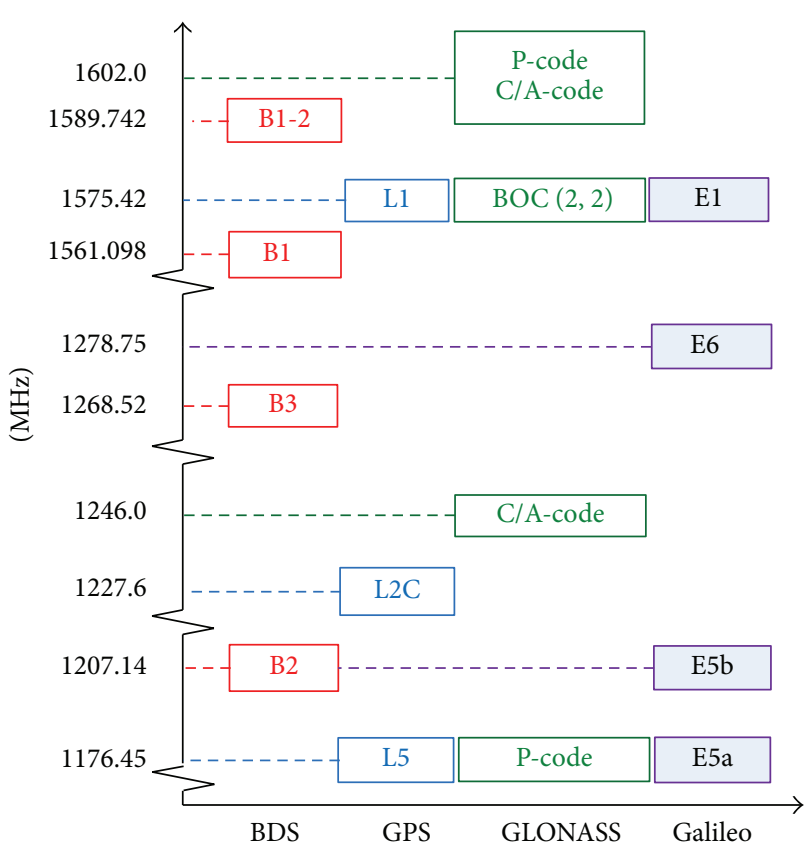

FIGURE 2: Spectrum distribution diagram of GNSS.

In Section 5, the GNSS signal receiving antennas of GEO, IGSO, and MEO BDS satellites are designed and conclusions are provided in Section 6.

\section{Spectrum Selection and Isolation Analysis}

In Section 2, the appropriate receivable signals are chosen, and the isolation budget is calculated.

According to the interface control documents (ICD) of GPS ICD 200 [6], GLONASS ICD [7], Galileo_ICD_2010 [8], and BDS ICD [9], the level of spectrum overlapping for different navigation system is high. The signal frequency chosen for BDS satellites orbit determination should be different from the transmitting signal frequencies on BDS-B1, BDS$\mathrm{B} 2$, and BDS-B3 bands in order to avoid the electromagnetic interference. Hence, the bandwidth of the signal receiving channel at the receiver should be narrowed in order to ensure the band suppression by filtering the out-of-band interference. The spectrum distribution diagram is shown in Figure 2.

As shown in Figure 2, among all the GNSS downlink signals, the signals carried on L2 band, including GPS L2-C and GLONASS C/A signals, are far from the BDS-B1, BDSB2, and BDS-B3 bands; hence, GPS L2C-BPSK(1) signal and GLONASS C/A Code-BPSK(0.511) signal are most appropriate for receiving the BDS satellites' orbit determination. These two signals are narrowband, which are $20.46 \mathrm{MHz}$ away from BDS-B2 band and $22.52 \mathrm{MHz}$ away from center frequency of BDS-B3 band. According to [10], QZSS satellites of Japan will also broadcast a signal on the same band of GPS L2 to enhance the open signal, and it will benefit the double signals receiving. Since these bands have been used by the GPS and GLONASS, other systems such as IRNSS and SBAS will not occupy these bands. The analyses of the electromagnetic 
TABLE 1: Beam coverage of BDS satellites' antenna.

\begin{tabular}{lcccc}
\hline Orbits & $H[\mathrm{~m}]$ & $\theta_{\mathrm{t}}[\mathrm{deg}]$ & $\theta_{\mathrm{s}}[\mathrm{deg}]$ & $\theta_{\mathrm{r}}[\mathrm{deg}]$ \\
\hline MEO & 21528000 & \pm 13.5 & \pm 13.2 & $>13.2$ or $<-13.2$ \\
IGSO & 35786000 & \pm 9.5 & \pm 8.7 & $>8.7$ or $<-8.7$ \\
GEO & 35786000 & \pm 9.5 & \pm 8.7 & $>8.7$ or $<-8.7$ \\
\hline
\end{tabular}

leakage and shielding effectiveness of the BDS satellites are given below.

The BDS satellites' downlink signal power is about $20 \mathrm{dBW}$ (equal to $50 \mathrm{dBm}$ ) [9] which is strongly amplified and directed toward the earth, while the downlink signals transmitted from other GNSS satellites on the other side of the earth are so weak and submerged in the noise. Therefore, the low noise amplifier (LNA) operates in saturation mode if the transmitted BDS signal leaks into its receiving channel. The nonlinear effect caused by the LNA prevents the receiver from acquiring and tracking the normal GNSS signals. Therefore, the budget of leaked transmitted signal power $\left(P_{1}\right)$ is calculated as

$$
P_{1}=P_{\mathrm{t}}-L_{\mathrm{s}}-L_{\mathrm{c}}-L_{\mathrm{bpf}}=-130 \mathrm{dBm},
$$

where $L_{\mathrm{s}}$ is the electromagnetism shielding efficiency, which is about $110 \mathrm{~dB}$ for general cable and radio frequency components [10]; $L_{c}$ is the coupling loss of the leaked transmitted signal from the space to the receiving antenna, which may be as low as $40 \mathrm{~dB}$ if the antenna installing position and direction are well tuned; $L_{\mathrm{bpf}}$ is the band suppression of band pass filter (BPF) and when the signal is out-of-band, it can be up to $40 \mathrm{~dB}$ with a well-designed narrowband BPF. Then $P_{1}$ of BDS is approximate to the received GNSS signal power: $P_{\mathrm{r}}=-175 \mathrm{dBW}=-145 \mathrm{dBm}$. Thus, by choosing the $\mathrm{L} 2$ band downlink signals of GPS and GLONASS, the receiver can suffer from the B2 and B3 signals from BDS satellites themselves.

\section{Signal Receiving Performance Analysis}

In Section 3, the signal receiving performance is analyzed by evaluating the Doppler frequency shift $\left(f_{\mathrm{d}}\right)$, the Doppler changing rate $\left(d f_{\mathrm{d}} / d t\right)$, the minimum number of visible satellites (MNVS), the carrier-to-noise ratio $\left(C / N_{0}\right)$, the GDOP, and the geometric distance root-mean-square (gdrms).

According to the geometrical relations of BDS satellites and the earth in the aerospace, the space is shaded by the earth on the range of $\pm 13.2 \mathrm{deg}$ from the satellite on $\mathrm{MEO}$, as the blue section in Figure 1. And it is $\pm 8.7 \mathrm{deg}$ from the satellite on GEO or IGSO. Then the designed transmitting antenna beam widths are $\pm 13.5 \mathrm{deg}, \pm 9.5 \mathrm{deg}$, and $\pm 9.5 \mathrm{deg}$ for BDS MEO, IGSO, and GEO satellites, respectively. Therefore, the range of available receiving beamwidth $\left(\theta_{\mathrm{r}}\right)$ can be obtained as shown in Table 1, where $H$ is the orbit altitude, $\theta_{t}$ is the transmitted downlink signal beamwidth, and $\theta_{\text {s }}$ is the earth shielding angle. In the available beamwidth ranges, the number of visible satellites (NVS), $f_{\mathrm{d}}, d f_{\mathrm{d}} / d t, C / N_{0}$, GDOP, and gdrms changing trends are analyzed in this section.

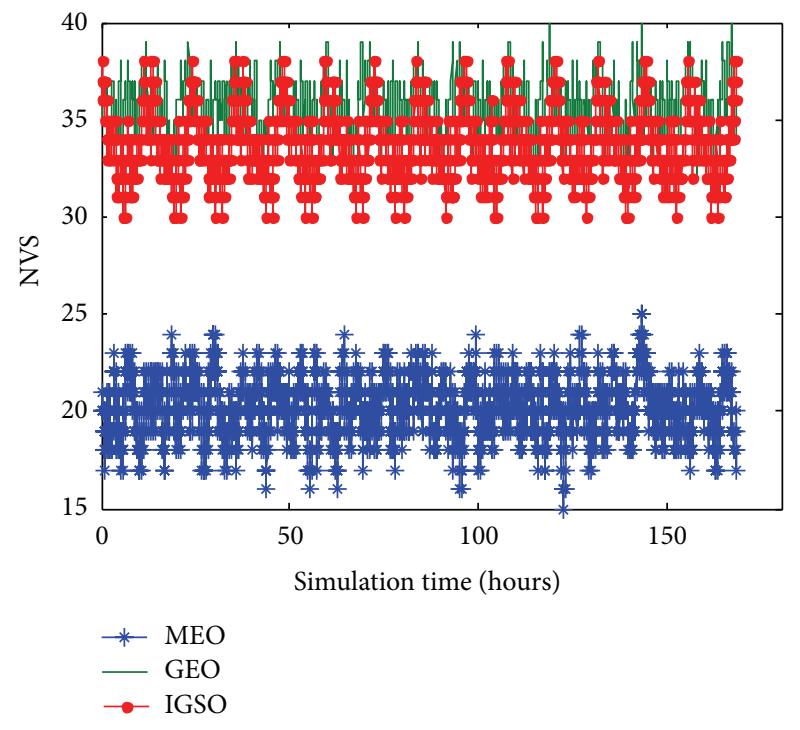

FIGURE 3: NVS changing trend from BDS MEO, GEO, and IGSO satellites.

3.1. Number of Visible Satellites (NVS). NVS is defined as the number of visible satellites during the whole time with the constraints on beam's starting elevation $\left(\theta_{0}\right)$ (unit: deg), beamwidth $\delta \theta$ (unit: deg), and $C / N_{0}$. Based on the definition of ICD files and ephemeris of GPS and GLONASS, the simulation model is generated with 24 GPS satellites and 24 GLNOASS satellites. The simulation runs for 7 days and Figure 3 illustrates the NVS performance of the BDS's MEO, GEO, and IGSO satellites.

It is revealed in Figure 3 that the NVS performance satisfies the demand of BDS satellite positioning on MEO, GEO, and IGSO during the whole simulation time without considering the beamwidth $(\delta \theta)$ and $C / N_{0}$ threshold of satellite's receiving antenna. The altitudes of the GEO and the IGSO are higher than that of the MEO; hence, the NVS observed by the GEO or IGSO satellites is higher. Specifically, the average NVS is $35.7,33$, and 20.1 on GEO, IGSO, and MEO, respectively.

In order to acquire the unambiguous positions of the satellite, the NVS should not be smaller than 4 [11]. In the following, we will evaluate the minimum number of visible satellites defined as minimum NVS (MNVS).

3.2. Doppler Frequency Shift $\left(f_{\mathrm{d}}\right)$ and Doppler Changing Rate $\left(d f_{\mathrm{d}} / d t\right)$. The 1st- and 2nd-order dynamics of the satellites result in drastic Doppler frequency shift $\left(f_{\mathrm{d}}\right)$ and Doppler changing rate $\left(d f_{\mathrm{d}} / d t\right)$. The ranges of the dynamic property should be calculated for evaluating the demands BDS satellites borne receiver.

For BDS satellites on MEO, GEO, and IGSO, $f_{\mathrm{d}}$ of received GPS and GLNOASS signals are simulated and shown in Figure 4. The first 24 hours' simulation results are given as an example.

The ranges of $f_{\mathrm{d}}$ on IGSO and MEO are more violent than that on GEO, because the GEO satellite is relatively static to the earth so that the relative speed towards GPS and 


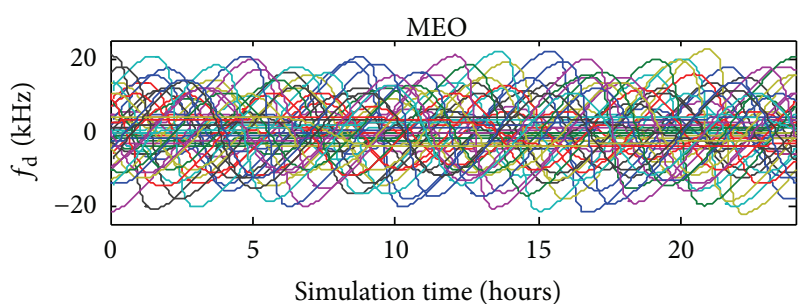

GEO
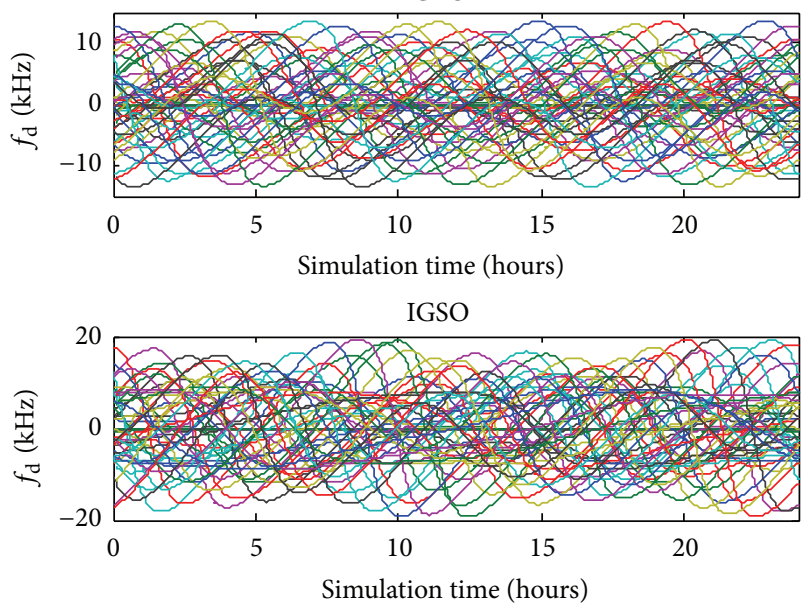

Figure 4: $f_{\mathrm{d}}$ of GLNOASS and GPS signals received on BDS satellites.

GLONASS satellites is also less than that of the BDS MEO or IGSO satellites.

The simulation results of $d f_{\mathrm{d}} / d t$ on BDS's MEO, GEO, and IGSO satellites are shown in Figure 5.

As shown in Figure 5 , most $d f_{\mathrm{d}} / d t$ values are negative, which indicates the fact that the parting velocity is larger than the approach velocity in most of time. The GNSS signal's $d f_{\mathrm{d}} / d t$ received on BDS MEO satellite varies sharply. The largest $d f_{\mathrm{d}} / d t$ observed on MEO satellites is approximately $-30 \mathrm{~Hz} / \mathrm{s}$ since $\mathrm{MEO}$ has the highest dynamic. But most $d f_{\mathrm{d}} / d t$ values fall within the range of $-5 \mathrm{~Hz} / \mathrm{s}$ to $3 \mathrm{~Hz} / \mathrm{s}$ on $\mathrm{MEO}$; from $-2 \mathrm{~Hz} / \mathrm{s}$ to $2 \mathrm{~Hz} / \mathrm{s}$ on $\mathrm{GEO}$, and from $-3 \mathrm{~Hz} / \mathrm{s}$ to $3 \mathrm{~Hz} / \mathrm{s}$ on IGSO.

According to [11], the acceptable $f_{\mathrm{d}}$ is at least $\pm 20 \mathrm{kHz}$ for a high dynamic receiver and the acceptable $d f_{\mathrm{d}} / d t$ is at least $\pm 10 \mathrm{~Hz} / \mathrm{s}$. By taking them as the Doppler tracking threshold and the Doppler changing rate threshold, spaceborne GPS and GLONASS receivers are feasible for the dynamic calculated above.

3.3. Carrier-to-Noise Ratio $\left(C / N_{0}\right)$. Without considering antenna gain's difference in different directions, the $C / N_{0}$ (unit: $\mathrm{dB}-\mathrm{Hz}$ ) can be calculated according to the receiver's $\left[G_{\mathrm{r}} / T\right]_{\mathrm{dB}}$ value (unit: $\mathrm{dB} / \mathrm{K}$ ) and the receiving power $P_{\mathrm{r}}$ (unit: $\mathrm{dBm})$ :

$$
\frac{C}{N_{0}}=P_{\mathrm{EIRP}}-L_{\mathrm{d}}+G_{\mathrm{r}}+\left[\frac{G_{\mathrm{r}}}{T}\right]_{\mathrm{dB}}-k,
$$

where $k=1.38^{-23} \mathrm{~J} / \mathrm{K}$ is the Boltzmann constant and $G_{\mathrm{r}}$ is the receiving antenna's gain (unit: $\mathrm{dB}$ ). $P_{\mathrm{EIRP}}$ is the equivalent
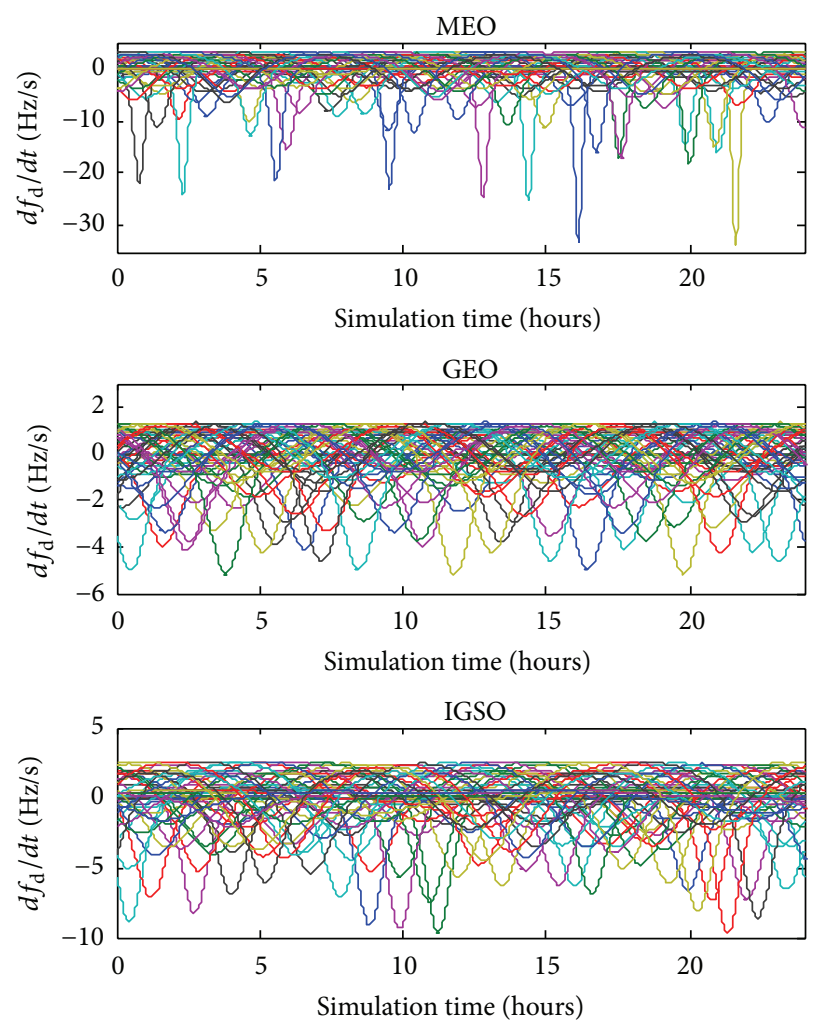

FIGURE 5: The $d f_{\mathrm{d}} / d t$ of GPS and GLNOASS signals observed from BDS's satellites.

isotropically radiated power (unit: $\mathrm{dBm}$ ) of the transmitter, and the free space loss (unit: $\mathrm{dB}$ ) $L_{\mathrm{d}}$ is expressed as

$$
L_{\mathrm{d}}=20 \lg \left(\frac{\lambda}{4 \pi R}\right),
$$

where $\lambda$ is the carrier wavelength (unit: $\mathrm{m}$ ) and $R$ is the distance between the GNSS satellites and the BDS satellites (unit: $\mathrm{m}$ ).

$G_{\mathrm{r}}$ is assumed to be $0 \mathrm{~dB}$ in this section for simplifying the understanding of the $C / N_{0}$ variations. The $C / N_{0}$ of GPS and GLONASS signals received on BDS MEO, GEO, and IGSO satellites are shown in Figure 6.

As shown in Figure 6 , the $C / N_{0}$ of the signals received on $\mathrm{MEO}$ is largest since the BDS MEO satellites are nearest to the GPS and the GLONASS satellites, which are also operating on MEO. The $C / N_{0}$ on BDS GEO and IGSO are close as they have similar altitudes.

Actually, the directional transmitting downlink antenna has a main lobe toward the earth and a side lobe toward the space around the earth. According to [12], the gain of transmitting antenna pattern may be obtained, as shown in Figure 7.

Compared with the main lobe gain of the GPS downlink antenna, the gain attenuation of the side lobe is about $15 \mathrm{~dB}$. The $3 \mathrm{~dB}$ beamwidth of its main lobe defined by GPS approaches the off axis angle relative to nadir. The signals transmitted from the side lobe are defined in the space service volume (SSV) of GPS ICD file where the lowest GPS III signal 


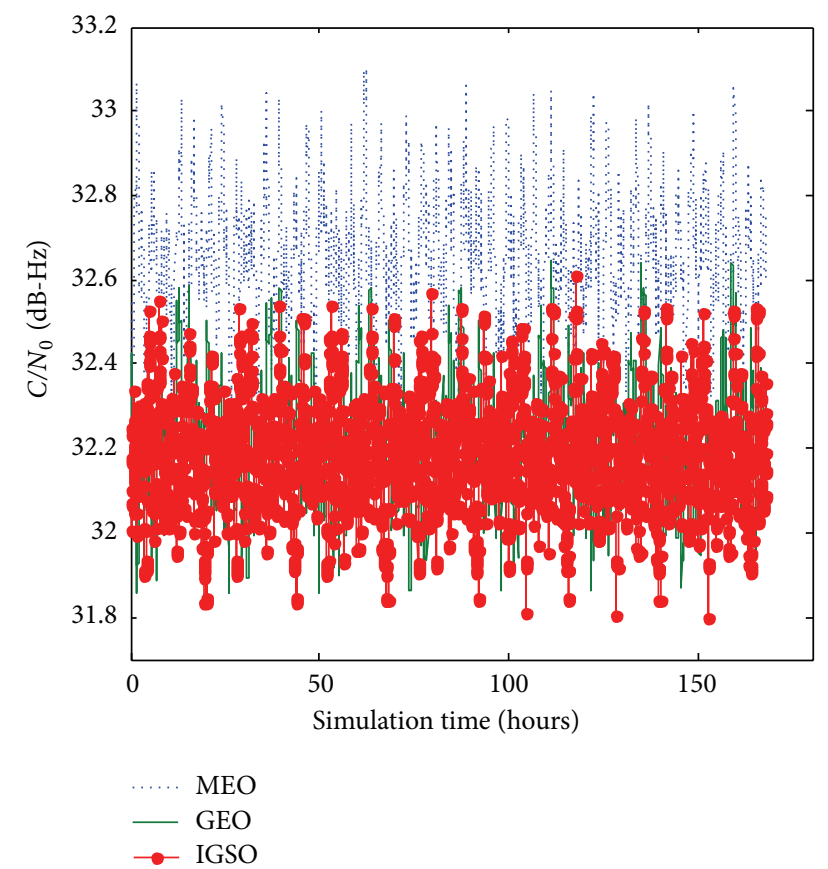

Figure 6: $C / N_{0}$ of GPS and GLONASS signals received on BDS satellites.

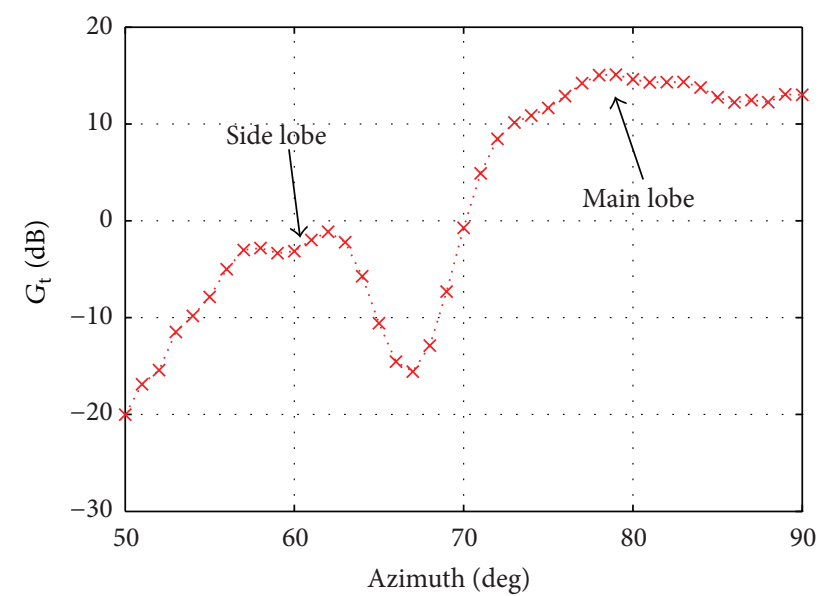

FIGURE 7: The downlink antenna pattern of GPS satellite (90 deg elevation).

powers received on $\mathrm{GEO}$ are $-186.0 \mathrm{dBW}$ for the $\mathrm{P}(\mathrm{Y})$ code and $-183.0 \mathrm{dBW}$ for the $\mathrm{C} / \mathrm{A}$ code.

When the receiver and transmitter are located on different sides of the earth, the receivers may be within the pattern's coverage of transmitting antenna. When the signal receiving $C / N_{0}$ is above the threshold, the GPS and GLONASS signals may be received by BDS satellites-borne receivers. The receiving gain $G_{\mathrm{r}}$ varies with the antenna's configurations read

$$
\left[G_{\mathrm{r}}\right]_{\mathrm{dB}}=\left[10 \lg \left[\left(\frac{\pi D}{\lambda}\right)^{2} \eta\right]\right]_{\mathrm{dB}},
$$

where $\eta$ is the antenna efficiency and $\eta \approx 50 \%$ in GPS. The approximate receiving antenna beamwidth $(\delta \theta)$ may be expressed as

$$
\delta \theta \approx 52 \frac{\lambda}{D}
$$

where $D$ is antenna's aperture (unit: $\mathrm{m}$ ). It is beneficial to improve $C / N_{0}$ by increasing the receiving gain of the antenna. However, a larger $G_{\mathrm{r}}$ may result in a smaller $\delta \theta$, which degrades the NVS performance. Therefore, the tradeoff between $\delta \theta$ and the $G_{\mathrm{r}}$ should be carefully struck, as we will show below.

The transmitting signals from GPS and GLONASS are designed and operated by other governments so that they cannot be changed by users. We can only change the receiving antenna on BDS in the future, to optimal $C / N_{0}$ and the signal tracking periods. Hence, the constraint of $C / N_{0}$ is necessary to be proposed, and by simulating with different $\delta \theta$, the optimal value can be sought out.

3.4. Dilution of Precision (GDOP). The GDOP value represents the deviation's amplification of the measurement's error attributed to solution position equations. GDOP is related to the constellation geometrical configuration and the receiver position. The GDOP may be readily calculated according to [13]. The formulas are left out here.

3.5. United Constraint of Geometric Distance Root-MeanSquare ( $g d r m s)$. A comprehensive factor is necessary for capturing the joint effects of MNVS, $C / N_{0}$, and GDOP on the received satellite signal quality. The authors of [11] adopted distance root-mean-square (drms) to measure the horizontal error in topocentric coordinate system. Motivated by [11], we focus on space positioning and proposed gdrms as the unified metric considering both the GDOP and the $\sigma_{\mathrm{UERE}}$ :

$$
\text { gdrms }=\text { GDOP } \times \sigma_{\mathrm{UERE}}
$$

where $\sigma_{\mathrm{UERE}}$ is given as

$$
\sigma_{\mathrm{UERE}}=\sqrt{\sigma_{\mathrm{t}}^{2}+\sigma_{\mathrm{e}}^{2}+\sigma_{\mathrm{inph}}^{2}+\sigma_{\mathrm{eph}}^{2}+\sigma_{\mathrm{rec}}^{2}+\sigma_{\mathrm{mult}}^{2}},
$$

where $\sigma_{\mathrm{t}}$ is the clock broadcast error; $\sigma_{\mathrm{e}}$ is the ephemeris broadcast error; $\sigma_{\text {rec }}$ is the receiver's noise resolution error; $\sigma_{\text {mult }}$ is the error from multipath effect (all units: $m$ ). in the spaceborne GNSS receiver, the effects of ionosphere and troposphere, which $\sigma_{\text {inph }}$ and $\sigma_{\text {eph }}$ are not exist. The typical values are shown in Table 2 [11].

The typical binary phase shift keying (BPSK) coarse acquisition (C/A) code receiver adopts an incoherent earlylate power mode delay lock loop (DLL) discriminator. $\sigma_{\text {rec }}$ can be calculated according to the actual code tracking accuracy: 


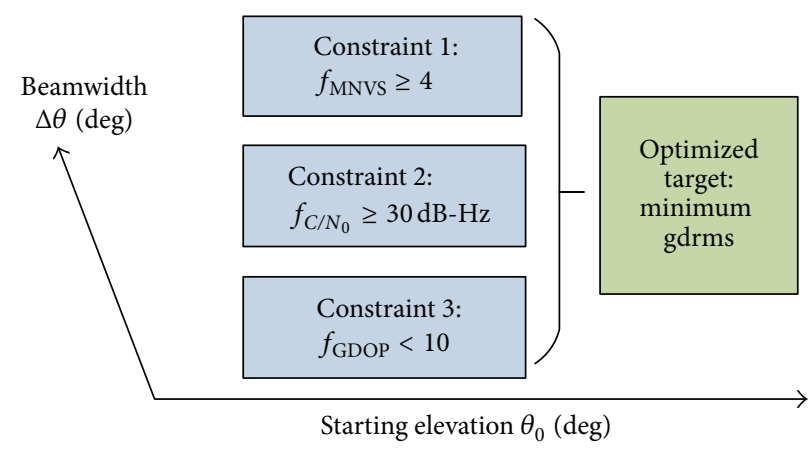

FIGURE 8: Flowchart of simulation and analysis.

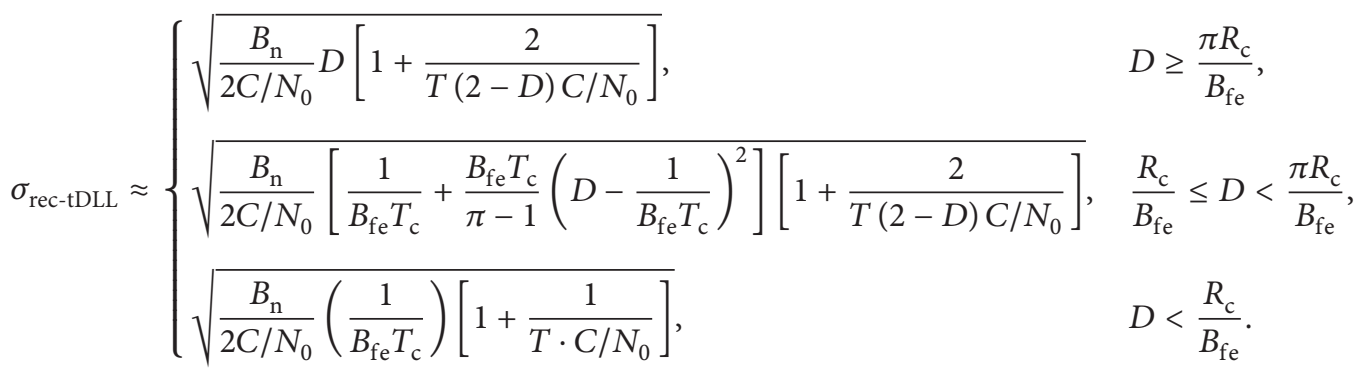

The meanings and typical values of the parameters are shown in Table 3. According to those formulas above, the unified gdrms may be chosen as the optimized objective in the optimal receiving antenna design.

\section{Simulation and Parameters Optimization}

The minimum gdrms is achieved by numerical simulations for the application. Since gdrms is determined by MNVS, $C / N_{0}$, and GDOP. The search space for MNVS, $C / N_{0}$, and GDOP are given in Figure 8, where the minimum gdrms is derived and the optimal antenna beam parameters may be acquired by numerical searching.

For GNSS signal receivers and the receiving antennas installed on MEO, GEO, and IGSO BDS satellites, the simulation is implemented by software STK and MATLAB with GPS and GLONASS's ephemeris for a BDS orbit's period of seven days.

4.1. Simulation with Constraint of MNVS. Taking MEO as an example, the interplay of the MNVS, $\theta_{0}$ (beam's starting elevation), and $\delta \theta$ (beamwidth) is analyzed by simulations. The ranges of $\left(\delta \theta+\theta_{0}\right) \leq 72$ deg within the GNSS transmitting antenna's coverage, while the normal direction is defined to $90 \mathrm{deg}$, and the tangential direction is defined to 0 deg.

When $\theta_{0} \in[0,72]$ and $\delta \theta \in\left[5,72-\theta_{0}\right]$, the MNVS of the GNSS signal received on BDS MEO satellite $\left(f_{\text {MNVS }}^{(\mathrm{MEO})}\left(\theta_{0}, \delta \theta\right)\right)$ is shown in Figure 9.

As shown in Figure 9, the maximum MNVS may be achieved in the range of $\theta_{0} \in[31,40]$ and $\delta \theta \in[29,41]$, where we have $\left|f_{\text {MNVS }}^{(\mathrm{MEO})}\left(\theta_{0}, \delta \theta\right)\right|_{\max }=13$. In the range of $\theta_{0} \in[0,52]$ and $\delta \theta \in[13,72]$, the constraints of $f_{\mathrm{MNVS}}^{(\mathrm{MEO})}\left(\theta_{0}, \delta \theta\right) \geq 4$ are satisfied.

Theoretically, increasing $\delta \theta$ and decreasing $\theta_{0}$ allows more satellites to fall into the receiver's antenna beam. However, the increasing $\delta \theta$ leads to the reduction of $C / N_{0}$. When the $C / N_{0}$ is below the threshold, it is unavailable for signal tracking and the MNVS is 0 . Therefore, the simulation above has considered the limitation of $C / N_{0}$ threshold.

4.2. Simulation with Constraint of $C / N_{0}$. Along with the constraint of MNVS, $C / N_{0}$ of GNSS signals are received on BDS MEO satellites where $f_{C / N_{0}}^{(\mathrm{MEO})}\left(\theta_{0}, \delta \theta\right)$ is in the ranges of $\theta_{0} \in[0,52]$ and $\delta \theta \in[13,72]$ obeying the constraint $f_{\mathrm{C} / N_{0}}^{(\mathrm{MEO})}\left(\theta_{0}, \delta \theta\right) \geq 30 \mathrm{~dB}-\mathrm{Hz} . \mathrm{C} / N_{0}$ of the received satellite signal for different $\theta_{0}$ and $\delta \theta$ is shown in Figure 10.

As shown in Figure 10, the maximum $C / N_{0}$ is achieved when $\theta_{0}=45 \mathrm{deg}$ and $\delta \theta=14 \mathrm{deg}$, where $\left|f_{\mathrm{C} / N_{0}}^{(\mathrm{MEO})}\left(\theta_{0}, \delta \theta\right)\right|_{\max }=50.63 \mathrm{~dB}-\mathrm{Hz}$.

4.3. Simulation with Constraint of GDOP. Combined with Constraint 1 and Constraint 2, the GDOP of GNSS signals are received by $\mathrm{BDS}$ satellite on $\mathrm{MEO}$ where $f_{\mathrm{GDOP}}\left(\theta_{0}, \delta \theta\right)$ is simulated by changing $\theta_{0}$ and $\delta \theta$. By obeying Constraint $3, f_{\mathrm{GDOP}}\left(\theta_{0}, \delta \theta\right)<10$. The variation of GDOP is shown in Figure 11 .

In order to simplify the illustration, the GDOP values are set to be 0 on conditions of $f_{\mathrm{GDOP}}^{(\mathrm{MEO})}\left(\theta_{0}, \delta \theta\right)>20$ or $f_{\mathrm{C} / N_{0}}^{(\mathrm{MEO})}\left(\theta_{0}, \delta \theta\right)<30 \mathrm{~dB}-\mathrm{Hz}$ or $f_{\mathrm{MNVS}}^{(\mathrm{MEO})}\left(\theta_{0}, \delta \theta\right)<4$. As 
TABLE 2: Typical URER budget for GPS.

\begin{tabular}{lcc}
\hline Segment & Error source & $1 \sigma$ error $[\mathrm{m}]$ \\
\hline Space/control & $\sigma_{\mathrm{t}}$ & 1.1 \\
& $\sigma_{\mathrm{e}}$ & 0.8 \\
\hline \multirow{3}{*}{ User } & $\sigma_{\text {inph }}$ & 0.1 (nonexistent here) \\
& $\sigma_{\text {eph }}$ & 0.2 (nonexistent here) \\
& $\sigma_{\text {rec }}$ & 0.1 (related to $\left.C / N_{0}\right)$ \\
Pseudo range error & $\sigma_{\text {mult }}$ & 0.2 \\
\hline
\end{tabular}

TABLE 3: Glossary list.

\begin{tabular}{lccl}
\hline Symbol & Typical value & Unit & Meaning \\
\hline$B_{\mathrm{n}}$ & 0.2 & $\mathrm{MHz}$ & Bandwidth \\
$D$ & 0.5 & chip & Early-late code correlator spacing \\
$T$ & 0.02 & $\mathrm{~s}$ & Preintegration time \\
$R_{\mathrm{c}}$ & 1.023 & $\mathrm{MHz}$ & Code rate \\
$B_{\mathrm{fe}}$ & $2 R_{\mathrm{c}}$ & $\mathrm{MHz}$ & Front-end bandwidth \\
$T_{\mathrm{c}}$ & $1 / R_{\mathrm{c}}$ & $\mathrm{s}$ & Code period \\
\hline
\end{tabular}

observed in Figure 11, the increase of $\delta \theta$ results in the decrease of GDOP on BDS MEO satellite. The changing trend of $f_{\mathrm{GDOP}}^{(\mathrm{MEO})}\left(\theta_{0}, \delta \theta\right)$ is inverse to $f_{C / N_{0}}^{(\mathrm{MEO})}\left(\theta_{0}, \delta \theta\right)$. According to the simulation, the valid ranges are limited to $\theta_{0} \in[0,52]$ and $\delta \theta \in[19,72]$. GDOP values observed in these ranges meet "Constraint 3."

4.4. Simulation with Constraint of gdrms. For the BDS MEO satellite, the gdrms is calculated within the ranges of $\theta_{0} \in$ $[0,53]$ and $\delta \theta \in[19,72]$, as shown in Figure 12(a). Similarly, the gdrms of the BDS satellites on GEO and IGSO may also be simulated and optimized for the receiving antenna design. The gdrms of the received GNSS signal on the BDS GEO and IGSO satellites are illustrated in Figures 12(b) and 12(c).

As shown in Figure 12, the gdrms of MEO becomes the least on $\theta_{0}=40 \mathrm{deg}$ and $\delta \theta=33 \mathrm{deg}$ which is $\left[f_{\text {gdrms }}^{(\mathrm{MEO})}\left(\theta_{0}, \delta \theta\right)\right]_{\min }=f_{\text {gdrms }}^{(\mathrm{MEO})}(40,33)=5.6843 \mathrm{~m}$. The least gdrms from the BDS GEO satellite occurred at conditions of $\theta_{0}=10 \mathrm{deg}$ and $\delta \theta=54 \mathrm{deg}$. The least gdrms from the BDS IGSO satellite was achieved when $\theta_{0}=6 \mathrm{deg}$ and $\delta \theta=61 \mathrm{deg}$.

At the same time, $C / N_{0}$, GDOP, and MNVS values of the parameters are calculated in Table 4.

All the observed parameters satisfied the 3 constraints and meet the demand of BDS satellite positioning.

\section{Designs of BDS Satellites' Receiving Antennas}

With the help of simulating software, CST Microwave Studio, the GNSS signals receiving antennas on BDS MEO, GEO, and IGSO satellites can be designed and simulated according to the optimal antenna beamwidth $(\delta \theta)$ and starting elevation $\left(\theta_{0}\right)$ simulated before. The patterns of designed antennas are shown in Figure 13.

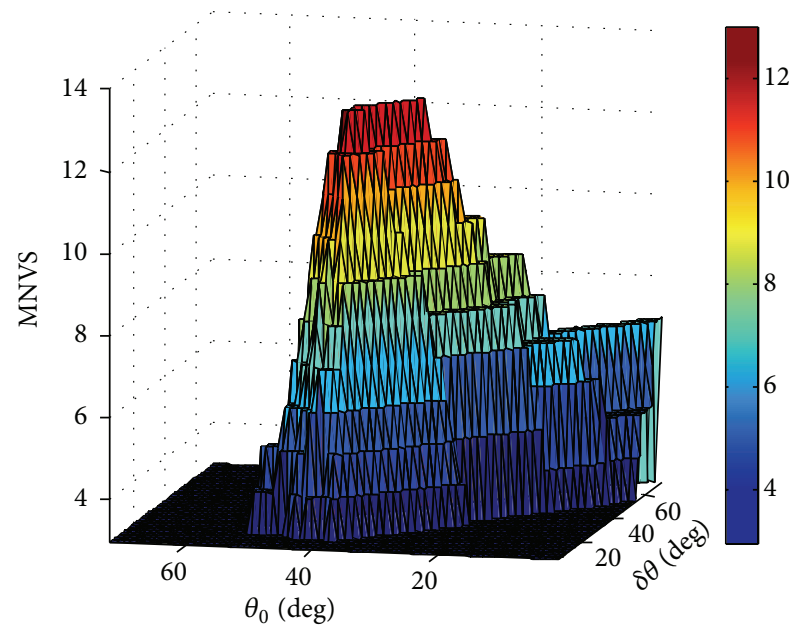

FIGURE 9: MNVS observed from MEO satellite.

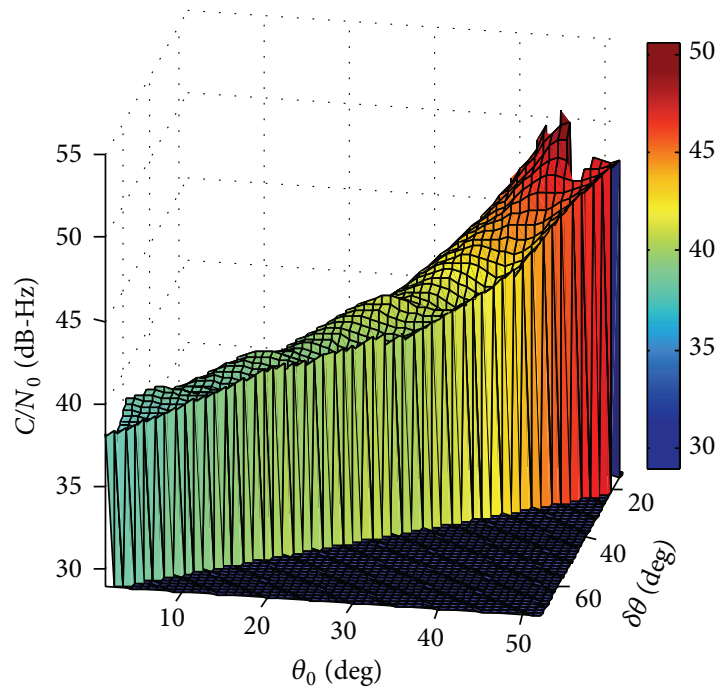

FIGURE 10: $C / N_{0}$ observed on MEO satellite.

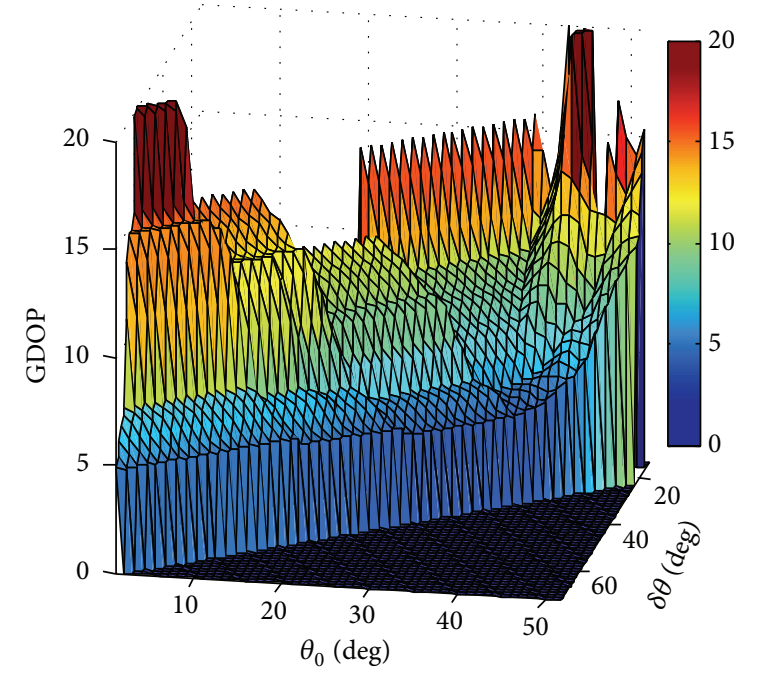

FIGURE 11: GDOP observed on MEO satellite. 


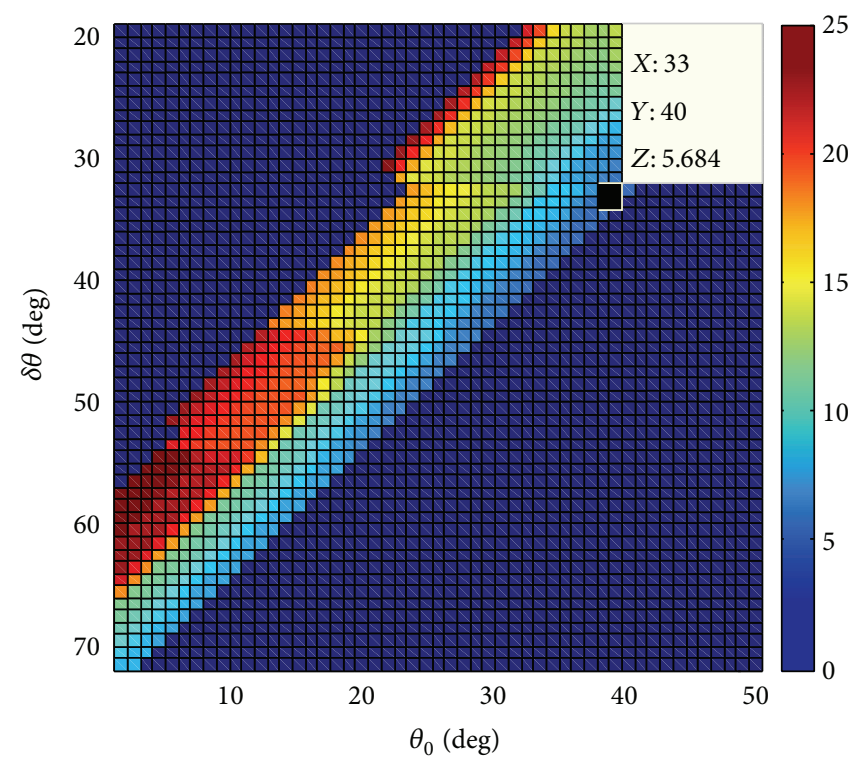

(a)

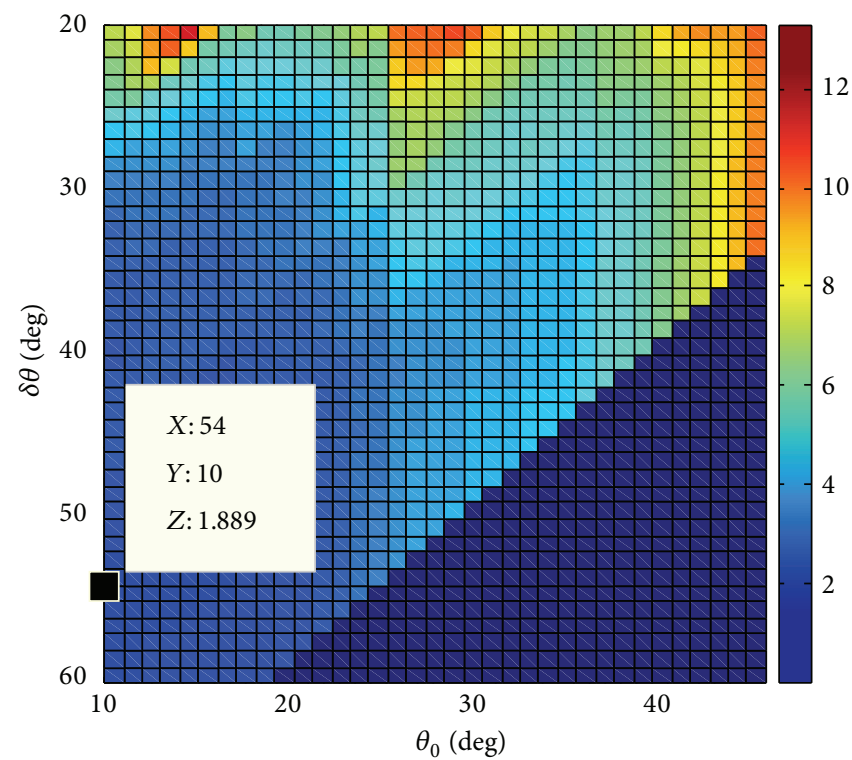

(b)

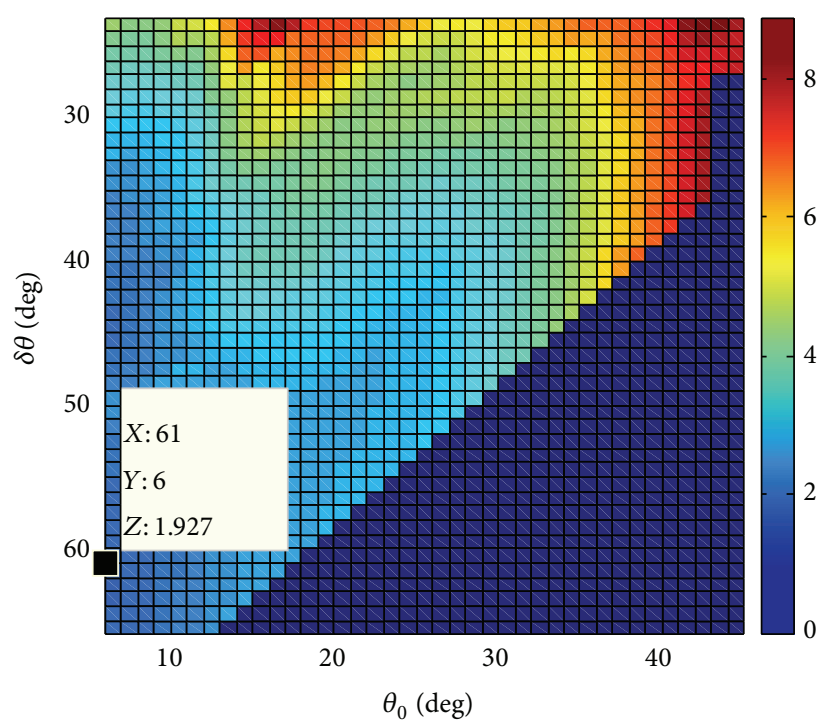

(c)

FIGURE 12: The gdrms changing trend of GNSS signal received from the BDS satellites. (a) Observed on MEO satellite. (b) Observed on GEO satellite. (c) Observed on IGSO satellite.

TABLE 4: Observations on BDS MEO, GEO, and IGSO satellites.

\begin{tabular}{lccc}
\hline Parameter & On MEO & On GEO & On IGSO \\
\hline gdrms $[\mathrm{m}]$ & 5.6843 & 1.8886 & 1.9266 \\
$C / N_{0}[\mathrm{~dB}-\mathrm{Hz}]$ & 42.6535 & 38.7638 & 38.7017 \\
GDOP & 3.9376 & 1.2126 & 1.2349 \\
MNVS & 13 & 19 & 19 \\
$\left(\theta_{0}, \delta \theta\right)[\mathrm{deg}]$ & $(40,33)$ & $(10,54)$ & $(6,61)$ \\
\hline
\end{tabular}

The beam of antenna on MEO satellite covers the range from $40 \mathrm{deg}$ to $73 \mathrm{deg}$ on elevation, while that on GEO and IGSO satellites covers the range from $10 \mathrm{deg}$ to $64 \mathrm{deg}$ and from $6 \mathrm{deg}$ to $67 \mathrm{deg}$, respectively. It is shown that the antenna on IGSO requires the largest beamwidth for covering enough satellites in order to obtain advanced performance.

As shown in Figure 13, the antennas patterns have lower gains in the direction towards the earth and form an annular beam in space. These designs make a larger $G_{\mathrm{r}}$ towards GNSS satellites in space around the earth than the general omnidirectional antenna. It may provide a better antijamming performance against the possible jamming signals coming from the earth.

\section{Summary}

In this paper, we propose novel orbit determination methods for BDS satellites with the aid of GPS and GLONASS signals. 


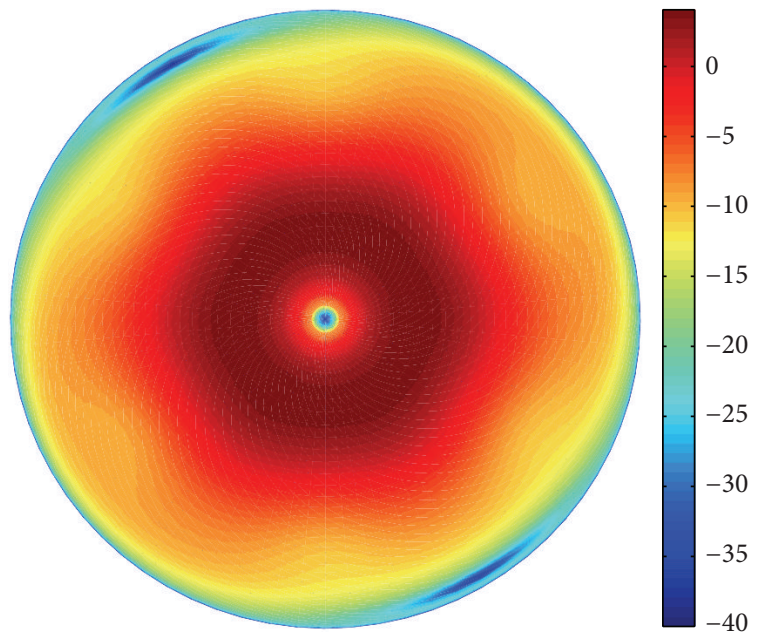

(a)

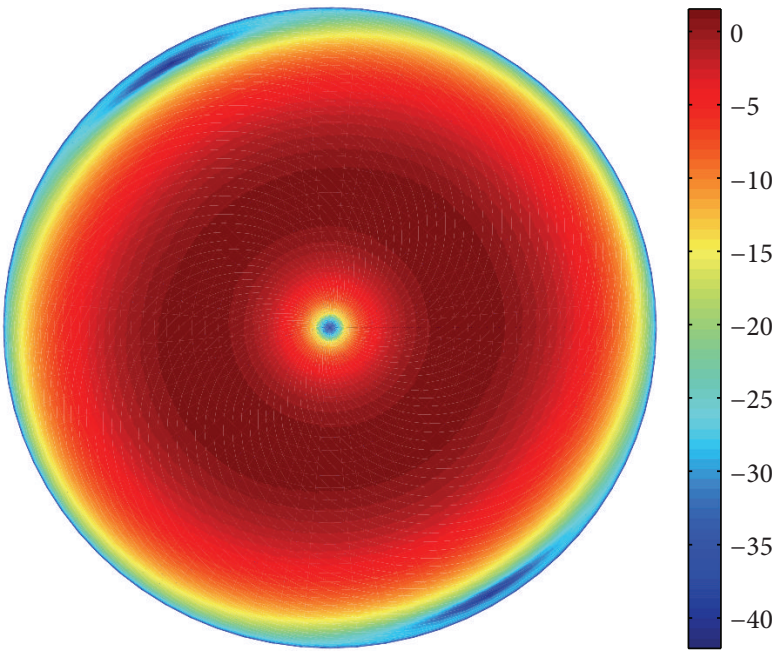

(b)

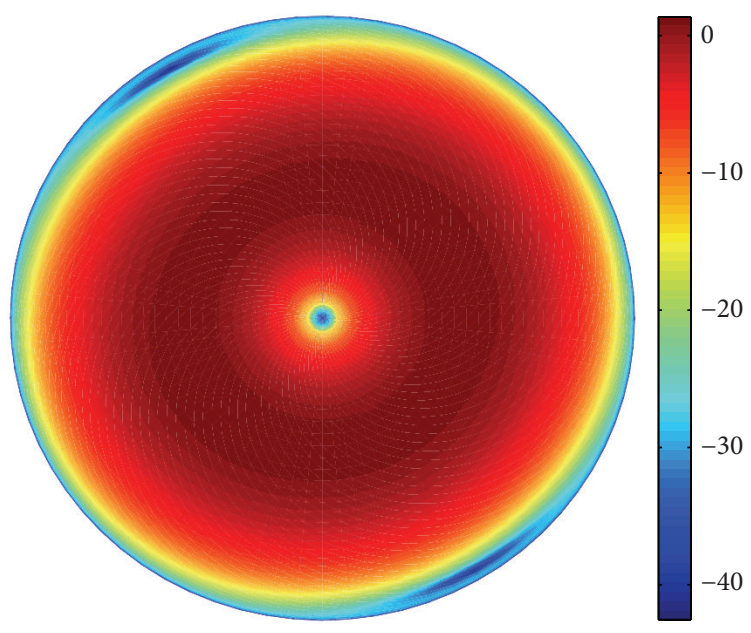

(c)

FIGURE 13: GNSS signal receiving antenna pattern on BDS satellites. (a) Antenna on MEO satellite. (b) Antenna on GEO satellite. (c) Antenna on IGSO satellite.

Two GPS and GLONASS signals on L2 band are chosen to avoid interference by the BDS's transmitting signals. By analysis and simulations, MNVS, $C / N_{0}$, GDOP, and gdrms are chosen as the evaluating parameters. The constraints have been determined for searching the optimal antenna performance. Then the optimal beamwidth $(\delta \theta)$ and starting elevation $\left(\theta_{0}\right)$ are obtained. With the optimization objective of minimum gdrms, BDS MEO satellites reach the minimum gdrms of $5.6843 \mathrm{~m}$ when the beam coverage is $40 \sim 73 \mathrm{deg}$ on elevation; BDS GEO satellites reach the minimum gdrms of $1.8886 \mathrm{~m}$ when the beam coverage is $10 \sim 64$ deg on elevation; BDS IGSO satellites reach the minimum gdrms of $1.9266 \mathrm{~m}$ when the beam coverage is $6 \sim 67 \mathrm{deg}$ on elevation. Finally, three antennas on BDS MEO, GEO, and IGSO satellites are designed. These contributions may be readily applied to the next generation BDS satellites.

\section{Competing Interests}

The authors declare that there is no conflict of interests regarding the publication of this paper.

\section{Acknowledgments}

The authors received financial aid from National Natural Science Foundation of China, Fund Code 41304026.

\section{References}

[1] C. Wen, M. Wang, L. Qi, D. Li, and Y. Qiu, “The application of the GNSS receiver in the third stage of china lunar exploration program," in Proceedings of the 27th Conference of Spacecraft TT\&C Technology in China, vol. 323 of Lecture Notes in Electrical Engineering, pp. 3-17, Springer, 2015.

[2] O. Balbach, B. Eissfeller, G. Hein, W. Enderle, M. Schmidhuber, and N. Lemke, "Tracking GPS above GPS satellite altitude: first results of the GPS experiment on the HEO mission equatorS," in Proceeedings of the IEEE Position Location and Navigation Symposium, pp. 243-249, Palm Springs, Calif, USA, April 1998.

[3] M. Moreau, F. Bauer, J. Carpenter, E. Davis, G. Davis, and L. Jackson, "Preliminary results from of the GPS flight experiment on the high earth orbit AMSAT OSCAR 40 spacecraft," in Proceedings of the 25th Annual AAS Rocky Montain Guidance and 
Control Conference, Guidance and Control, vol. 11 of Advance in the Astronautical Sciences, pp. 44-59, 2002.

[4] M. Christian and L. Denis, "Real-time GEO orbit determination using TOPSTAR 3000 GPS receiver," Navigation, vol. 48, no. 3 , pp. 169-179, 2001.

[5] L. Qiao, S. Lim, and J. Liu, "Autonomous GEO satellite navigation with multiple GNSS measurements," in Proceedings of the 22nd International Technical Meeting of the Satellite Division of the Institute of Navigation (ION GNSS '09), pp. 3611-3619, Savannah, Ga, USA, September 2009.

[6] GPS ICD 200, IS-GPS-200 Revision D, IRN-200D-001: NAVSTAR global positioning system Interface Specification, NAVSTAR GPS Space Segment/Navigation User Interface, USA, 2006, http://www.gps.gov/technical/icwg/IS-GPS-200D.pdf.

[7] V. A. Menshikov and G. M. Solovyev, "Global Navigation Satellite System (GLONASS)," in Van Nostrand's Scientific Encyclopedia, 2006.

[8] Galileo_ICD_2010, European GNSS (Galileo) open service: signal in space: interface control document. Publications Office of the European Union, Issue 1, European GNSS, 2010, http://w3.uch.edu.tw/ccchang50/Galileo_ICD_2010.pdf.

[9] BDS ICD, "BeiDou navigation satellite system signal in space interface control document," Open Service Signal Version 2.0. China Satellite Navigation Office, 2013.

[10] J. W. Betz, Signal structure for satellite-based navigation past, present, and future, Inside GNSS, 2013, http://www.insidegnss .com.

[11] E. Kaplan and C. Hegarty, Understanding GPS Principles and Application, Artech House, 2nd edition, 2008.

[12] W. Parkinson and J. Spilker, Global Positioning System: Theory and Applications, Volume II, American Institute of Aeronautics and Astronautics, 1996.

[13] L. Chen, Y. Huang, W. Liu, and G. Ou, "Feasibility analysis of GNSS multi-constellation positioning for lunar spacecraft," in China Satellite Navigation Conference (CSNC) 2015 Proceedings: Volume I, vol. 340 of Lecture Notes in Electrical Engineering, pp. 749-763, 2015. 


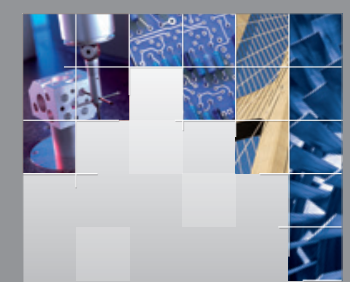

\section{Enfincering}
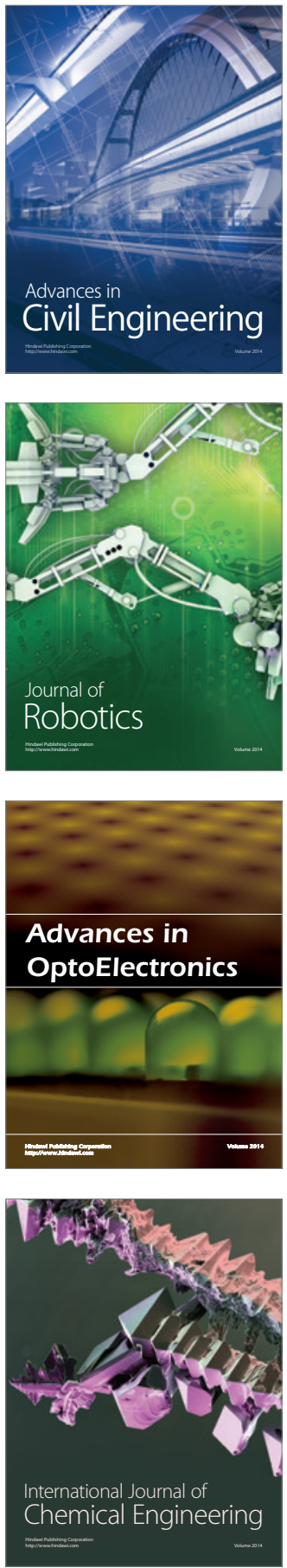

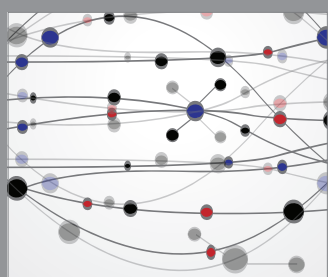

The Scientific World Journal

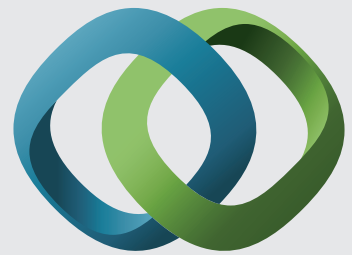

\section{Hindawi}

Submit your manuscripts at

http://www.hindawi.com
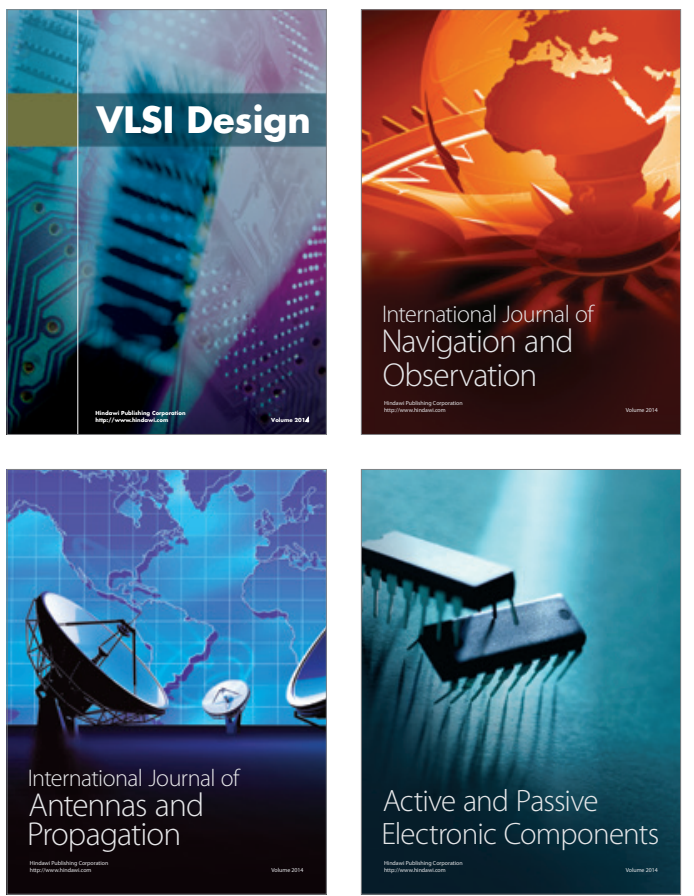
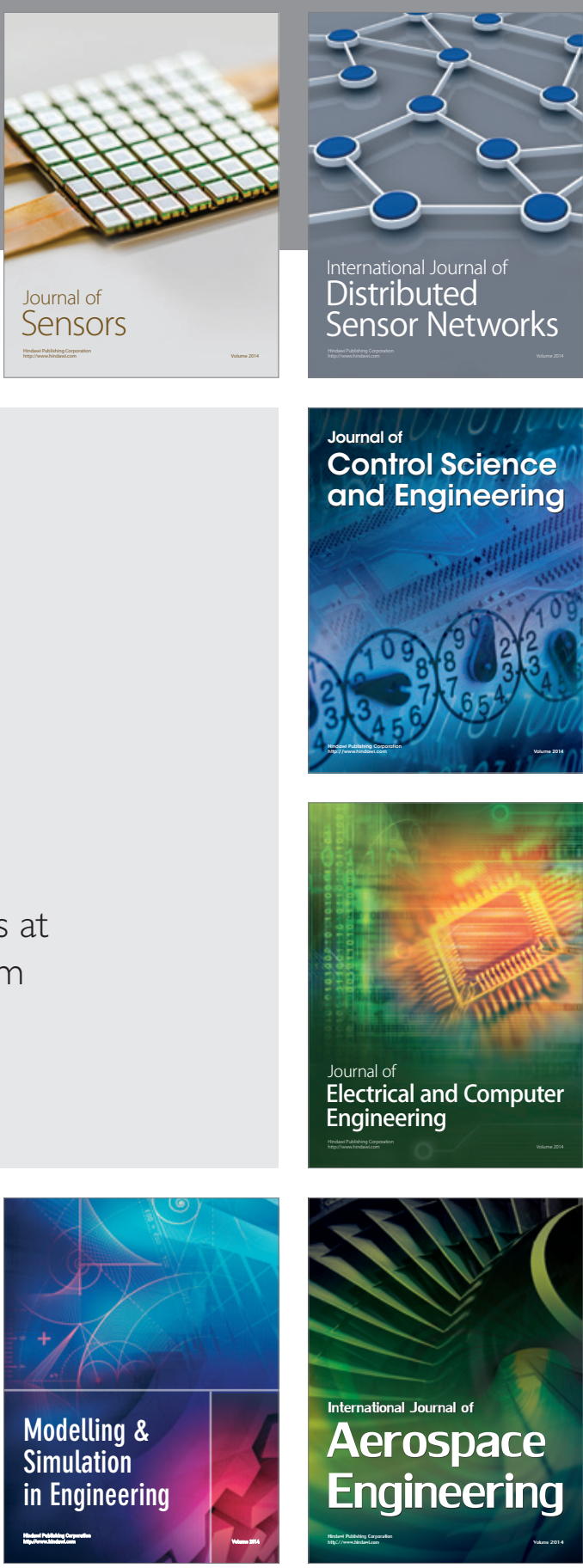

International Journal of

Distributed

Sensor Networks

Journal of

Control Science

and Engineering
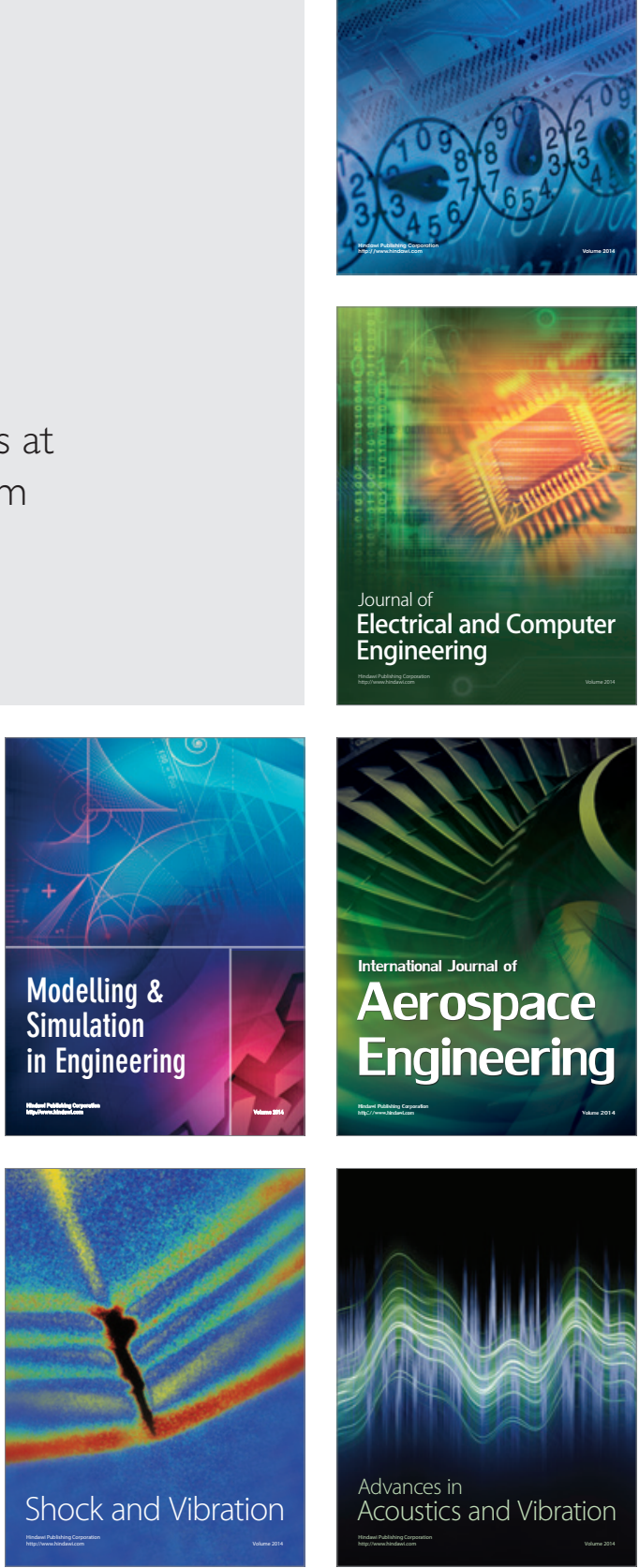